\title{
Mechanisms and Enhancement of Flame Stabilization
}

(Grant No. DE-FG02-89ER13988.A001)

DOE/ER/13988--T1

DE92 007911

Submitted by:

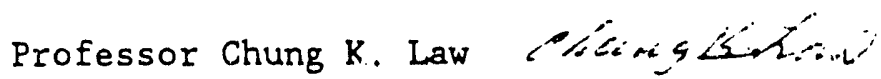

Department of Mechanical and Aerospace Engineering

Princeton University

Princeton, NJ 08544

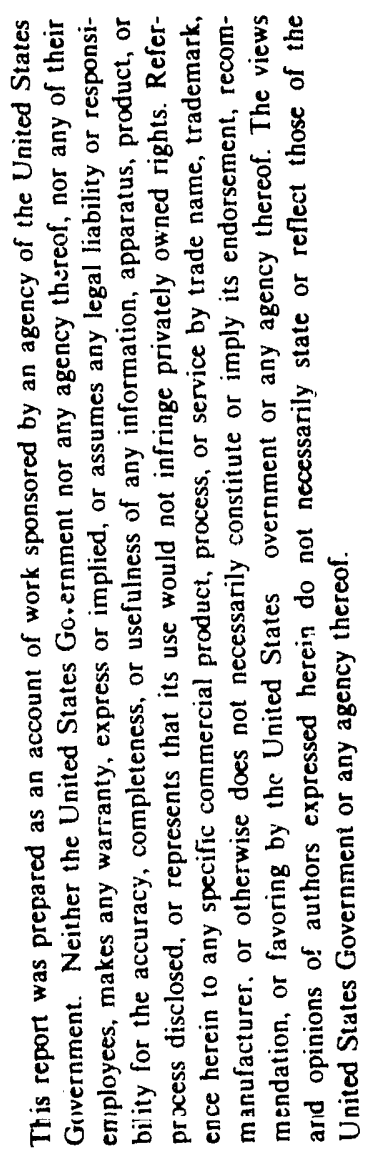

For Consideration by:

Dr. Daniel Frederick

Division of Engineering, Mathematics and Geosciences

Office of Basic Energy Sciences, ER-15

Department of Energy

Washington, DC 20544

Endorsed by:

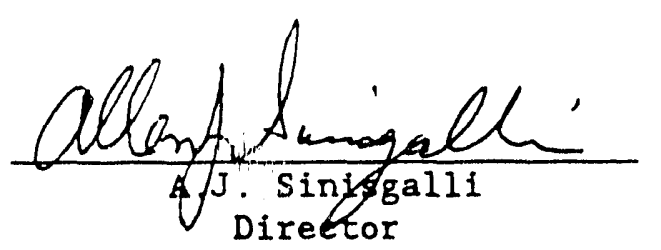

Office of Research and Projects Administration

Princeton University

July $i 0,1990$ 
During the reporting periud the following projects have been conducted.

1. Structure and Extinction of Diffusion Flames with Flame Radiation Using droplet combustion as a model problem, and capitalizing on the temperature-sensitive nature of radiative heat transfer, the structure and extinction of diffusion flames with flame radiation is studied via multiscale activation energy asymptotics. The flame structure analyzed consists of an $O(\epsilon)$ reaction zune embedded within an $O(\delta)$ radiation zone which in turn is situated in the $O(1)$ diffusive-convective flow field, where $\epsilon<<<<1$. The analysis yields the structure equation for the reaction zone, which can be cast in the same form as that of Liñan's adiabatic diffusion flame problem such that his extinction results can be readily used. Present results show that radiative heat loss promotes flame extinction in general, as expected. Furthermore, it can also lead to the phenomenon of dual extinction turning points in which flame extinction due to reactant leakage and thereby kinetic limitation occurs not only for sufficiently small droplets, as is well established, but also for sufficiently large droplets as a result of excessive heat loss from the correspondingly large flame. Consequently there exist diffusive-reactive-radiative systems for which steady combustion is not possible for all droplet sizes. An estimation of the dimensional radiative extinction droplet size is also given for the sample system studied.

This work is reported in Publication lio. 1. 


\section{Curvature Effects on Diffusion Flame Extinction}

Extinction of wrinkled diffusion flames has been studied via the model problem of the opening of the Burke-Schumann flame tip. Asymptotic analysis of the flame structure in the tip region shows that increasing the extent of the flame curvature facilitates near-complete reaction and thereby enhances the burning intensity. Consequently, for unity Lewis number flames, increases in the flow velocity tend to inhlbit tip opening because of the corresponding decrease in the radius of the flame tip. Experimental results using near-unity Lewis number acetylene/air flames agree with the predicted flame geometry and its inability to achieve tip opening. Tip opening, however, can be achieved by using a sub-unity Lewis number fuel stream of hydrogen and carbon dioxide, which causes a general lowering of flame temperature in the entire flame tip region. Further experiments then confirm the theoretical result that negative stretch, or compression, in the form of negative flame curvature promotes burning and thereby retards extinction.

This work is reported in Publication No. 2.

\section{A Kinetic Criterion of Flammability Limits}

An experimental and theoretical investigation has been conducted on the determination of the flammability limits of the $\mathrm{C}-\mathrm{H}-\mathrm{O}$-inert system and on the understanding of limit phenomena in general. Experimentally, flammability limits have been determined by first measuring the extinction limits of stretched, counterflow flames and extrapolating the results to zero stretch. Consequently, lean and rich flamability limits have been determined for mixtures of methane, ethane, ethylene, acetylene and propane with air, for mixtures of $\mathrm{CO}$ and $\mathrm{H}_{2}$, and for the effects of dilution, inert 
substitution, and chemical additives such as $\mathrm{CH}_{3} \mathrm{Br}$ and $\mathrm{H}_{2}$. By further hypothesizing that the limit phenomena are primarily controlled by the kinetic processes of chain branching versus termination, a predictive theory has been advanced for the a priori determination of flammability limits. Calculated results largely agree with the experimental data, for both the lean and rich limits. The study further shows that $\mathrm{H}+\mathrm{O}_{2} \rightarrow \mathrm{O}+\mathrm{OH}$ is the dominant termination reaction for all lean and rich limits, that $\mathrm{H}+\mathrm{O}_{2}+\mathrm{M} \rightarrow \mathrm{HO}_{2}+\mathrm{M}$ is the dominant termination reaction for all lean limits, that the dominant termination reaction for rich limits can be mixture specific, and that as the flammability limit is approached the maximum termination rate occurs in the same region as that of the maximum branching rate, thereby allowing for most efficient radical scavenging. Pressure effects on rich limits and the concept of limit temperature are also interpreted based on the present theory and understanding.

This work is reported in Publication No. 3.

\section{Development of Laser Diagnostic Techniques}

A major component of our program is to study the flame structure and the mechanism of flame stabilization by using laser probing. During the reporting period extensive amount of effort has been expended towards the development of spontaneous Raman spectroscopy and laser induced fluorescence techniques in order to determine the temperature and concentration profiles of flames. The laser system has been acquired and substantial progress has been made in its set-up. We expect to be able to take quantitative measurements during the next year. 
1. "Structure and Extinction of Diffusion Flames with Flame Radiation," by B.H. Chao, C.K. Law and J.S. Tien, to appear in Proc. of twenty-third Combustion Symposium.

2. "On the Opening of Barke-Schumann Flame Tip and the Effects of Curvature on Diffusion Flame Extinction," by H.G. Im, C.K. Law, and R.L. Axelbaum, to appear in Proc. of Twenty-Third Combustion Symposium.

3. "A Kinetic Criterion of Flammability Iimits: The C-H-O-Inert System," by C.K. Law and F.N. Egolfopoulos, to appear in Proc. of Twenty-Third Combustion Symposium. 

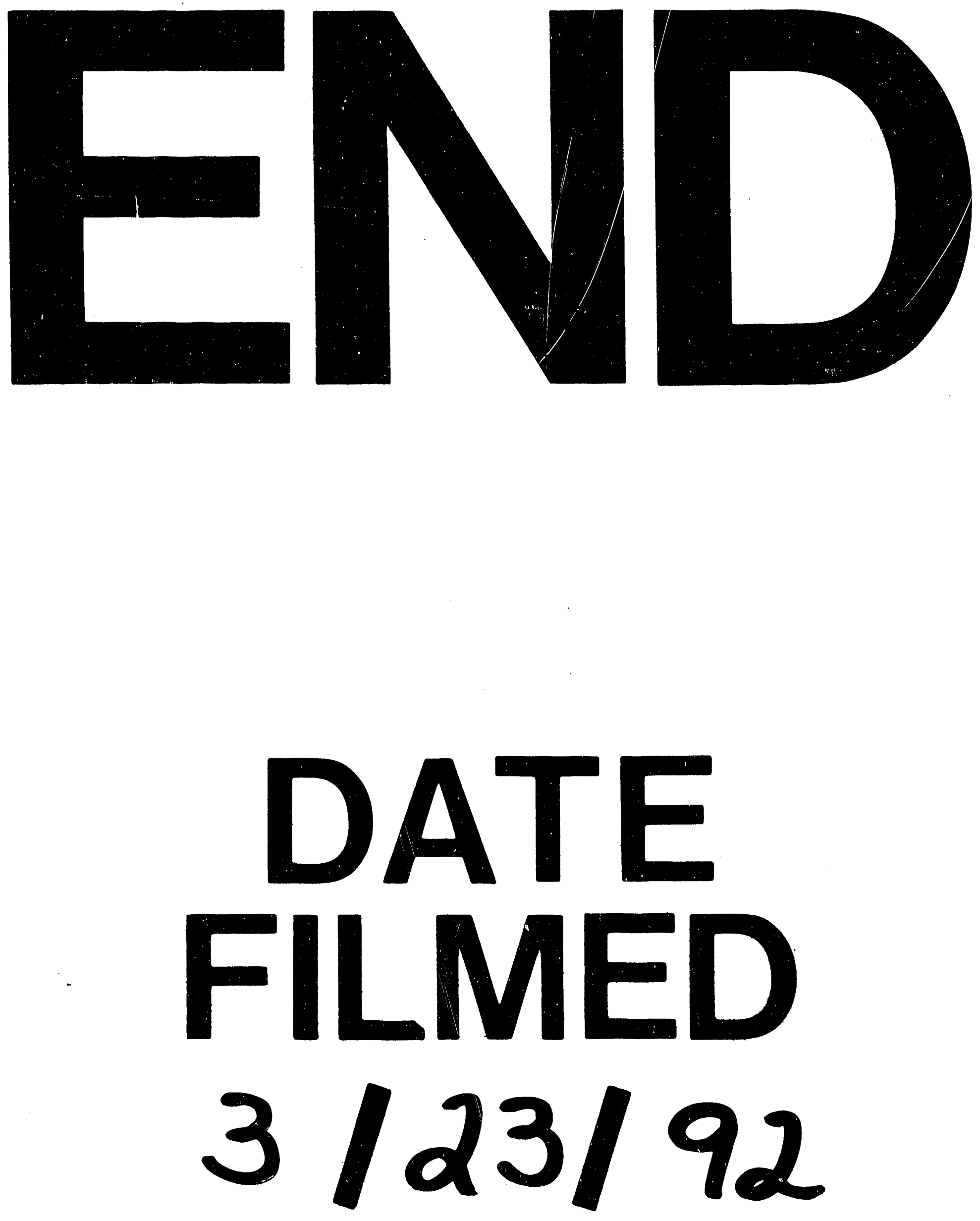

1 
rminnm

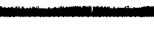

